(Aus dem physiologischen Laboratorium in Bonn.)

\title{
Ueber die von Kutscher und Steudel beobachtete Unsicherheit in der Methode der stickstoffbestimmung nach Kjeldahl.
}

Von

\section{Bernhard Sehöndorfr.}

In der Zeitschrift für physiologische Chemie Bd. 39 Heft 1 S. 12-21 veröffentlichen Kutscher und Steudel eine Untersuchung über die Anwendung der Kjeldahl'schen Stickstoffbestimmungsmethode bei verschiedenen für die Physiologie in Betracht kommenden stickstoffhaltigen Körpern.

Während es längst bekannt wäre, dass bei ganzen Gruppen von Körpern die Methode versagte, Nitro-, Cyan- u. s. w. Verbindungen liessen sich nicht oder doch nur nach gewissen Vorbereitungen nach Kjeldahl analysiren, so gelte es von sämmtlichen stickstoffhaltigen Verbindungen, die physiologisch in Betracht kämen, für feststehend, dass sie quantitativ ihren Stickstoff bei der Veraschung nach Kjeldahl als Ammoniak abgäben, und sie hätten in der äusserst umfangreichen Stoffwechselliteratur, in der alle erdenklichen Fragen behandelt würden, keine einzige Arbeit gefunden, in der dieses Fundamentaldogma der modernen Stoffwechselmethodik auch nur einem leichten $Z$ weifel begegnet wäre ${ }^{1}$ ).

$\mathrm{Kutscher}$ und Steudel fanden bei der Untersuchung verschiedener Amidsubstanzen, dass sie bei Anwendung der Kjeldahlschen Methode, indem sie bei der Oxydation zur Schwefelsäure Kupfersulfat und Kaliumpermanganat zusetzten, nicht allen Stickstoff als Ammoniak erhielten. Und zwar stellte sich nicht ein gleichmässiger Stickstoffverlust ein, sondern derselbe war verschieden gross.

1) Im Original nicht gesperrt gedruckt. 
Sie führten die Bestimmung so aus, dass sie 0,15-0,2 g Substanz mit $10 \mathrm{ccm}$ concentrirter Schwefelsäure und einem bohnengrossen Stück $\mathrm{CuSO}_{4}$ etwa 10 Minuten bis zum lebhaften Sieden erhitzten, erkalten liessen und dann mit einer Messerspitze Kaliumpermanganat oxydirten, wieder bis zum Sieden erhitzten und das Reactionsgemisch in bekannter Weise weiter behandelten.

Sie fanden z. B. bei der Untersuchung von Kreatin, dessen berechneter $\mathrm{N}$-Gehalt in krystallwasserhaltigem Kreatin $28,23 \% \mathrm{~N}$ beträgt:

$\begin{array}{lrrr}23,84 \% & \mathrm{~N} & \text { Verlust }-4,39 \%, \\ 24,63 \% \mathrm{~N} & " & -3,60 \%, \\ 22,23 \% \mathrm{~N} & " & -6,00 \%, \\ 27,31 \% \mathrm{~N} & " & -0,92 \%, \\ 26,10 \% \mathrm{~N} & & -2,13 \% .\end{array}$

Die Substanz, nach D umas analysirt, ergab $28,65 \%$, also diè theoretische Zahl.

Dann modificirten sie die Menge des zugesetzten $\mathrm{CuSO}_{4}$ oder liessen die Oxydation mit Kaliumpermanganat oder mit Kupfersulfat weg oder veränderten die Zeitdauer des Kochens; sie erhielten dann Differenzen bis $\mathrm{zu}-7,37 \%$, zuweilen aber auch den theoretisch berechneten Werth.

Die Untersuchung des Kreatinins nach Kjeldahl ergab Differenzen von $6,25 \%$ bis $16,06 \%$.

Auch bei der Untersuchung der Harnsäure ergaben sich, wenn auch nicht so grosse Unterschiede, aber doch Differenzen bis zu $1,37 \%$, zuweilen auch die theoretisch berechnete Menge.

Ebenso fanden sie auch bei der Untersuchung von Lysin und Histidin derartige grosse Stickstoffverluste.

Diese Beobachtungen stehen im Widerspruch mit Untersuchungen, die im hiesigen Institut schon vor vielen Jahren, von A rgutinsky ${ }^{1}$ ) im Jahre 1890 und von mir $^{2}$ ) im Jahre 1896, veröffentlicht wurden. Dieselben haben mit unzweifelhafter Sicherheit festgestellt, dass bei den fürdie Physiologie in Betracht kommenden Stickstoffverbindungendie Kjeldahl'sche

1) Dieses Archiv Bd. 46 S. 591.

2) Dieses Archiv Bd. 62 S. 1-57. 
Methode den gesammten Stickstoff ergibt, so dass also in der Literatur ein Zweifel an dieser Thatsache gar nicht gefunden werden konnte.

Wir bestimmten den Stickstoff derartig, dass wir die Substanz mit $25 \mathrm{ccm}$ concentrirter Schwefelsäure und $0,1 \mathrm{ccm}$ metallischen Quecksilbers längere Zeit, ca. 1 Stunde, wenn nöthig noch länger, kochten und nicht nur bis zum Sieden erwärmten und dann nach Zusatz von Natronlauge und Natriumsulfid das gebildete Ammoniak in eine vorgelegte, titrirte Schwefelsäure überdestillirten.

Nach Argutinsky's Untersuchung ergab auf diese Weise analysirt:

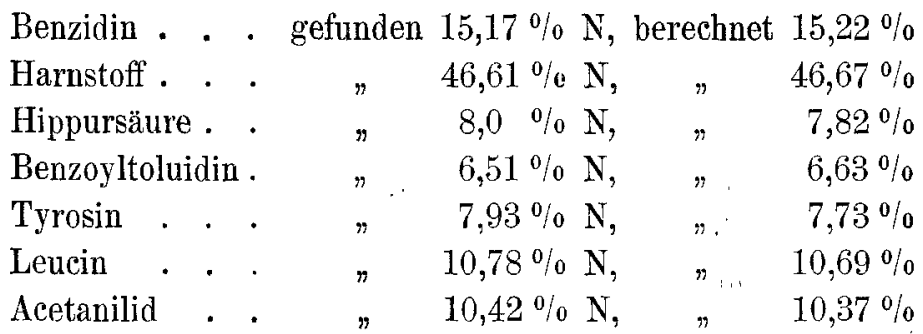

Ich habe dann später in einer systematischen Untersuchung die meisten der damals bekannten und für die Physiologie wichtigen stickstoffhaltigen Körper, soweit sie mir zugänglich waren, hinsichtlich der Anwendung der Kjeldahl'schen Methode untersucht und habe festgestellt, dass die Wilfarth'sche Modification der Kjeldahlschen Methode ausnahmslos die richtigen Werthe für den Stickstoff ergibt. Die Körper waren theils von mir selbst rein dargestellt, theils anderweitig erhalten und, soweit es möglich, durch die Schmelzpunktsbestimmung auf ihre Reinheit gepruft. Ich lasse im Folgenden einen Auszug aus der Generaltabelle meiner Arbeit folgen, der diese Thatsache mit absoluter Sicherheit beweist. Die zuweilen gefundenen Unterschiede liegen, absolut genommen, im Bereich des Beobachtungsfehlers, machen aber, procentisch gerechnet, eventuell einen grösseren Unterschied aus.

\begin{tabular}{|c|c|c|c|c|c|}
\hline Glykokoll & & gefunden & $18,53 \% \mathrm{~N}$, & berechnet & t $18,64^{\circ} \%$ \\
\hline Alanin & & $n$ & $15,72 \% \mathrm{~N}$, & $"$ & $15,73 \%$ \\
\hline Leucin & & $"$ & $10,48 \% \mathrm{~N}$, & $"$ & $10,69 \%$ \\
\hline Sarkosin & . & $n$ & $15,6 \% \mathrm{~N}$, & $n$ & $15,73 \%$ \\
\hline Taurin & . & $"$ & $11,07 \% \mathrm{~N}$ & $n$ & $11,2 \%$ \\
\hline
\end{tabular}


Tyrosin . . gefunden $7,75 \% \mathrm{~N}$, berechnet $7,73 \%$ m-Amidobenzoësäure " $10,12 \% \mathrm{~N}, \quad$ " $10,22 \%$ Asparaginsäure. $\quad " 10,48 \% \mathrm{~N}, \quad$ " $10,53 \%$ Harnsäure . . $\quad 33,03 \% \mathrm{~N}, \quad$ " $33,33 \%$ Allantoïn . . $\quad 35,26 \% \mathrm{~N}, \quad \cdots \quad 35,44 \%$ Alloxantin . . $\quad " 17,41 \% \mathrm{~N}, \quad " \quad 17,39 \%$ Kaffeïn . . $\quad \# 28,9 \% \mathrm{~N}, \quad \# \quad 28,87 \%$ Xanthin . . . $\quad$ " $36,35 \% \mathrm{~N}, \quad, \quad 36,84 \%$ (In der untersuchten Menge gefunden 7,0 $\mathrm{mg} \mathrm{N}$, berechnet 7,1 $\mathrm{mg} \mathrm{N}$.) Guanin . . . gefunden $46,303 \% \mathrm{~N}$, berechnet $46,36 \%$ Kreatinin . . $\quad 3 \quad 37,25 \% \mathrm{~N}, \quad$ $\quad 37,17 \%$ Kreatin (wasserfrei) $\quad 3 \quad 32,09 \% \mathrm{~N}, \quad$ \% $32,06 \%$.

Wie man deutlich sieht, ergibt die Untersuchung auch der Körper, bei welchen Kutscher und Steudel die grossen Verluste hatten, genau den theoretisch berechneten Werth. Körper wie Lysin, Histidin, Arginin u. s. w. standen mir damals nicht zur Verfügung. Da aber wenigstens bei Lysin und Arginin, deren Constitution bekannt, die Bindung des $\mathrm{N}$ in ähnlicher Weise stattfindet wie bei verschiedenen der oben untersuchten Körper, so ist wohl als sicher anzunehmen, dass auch bei diesen Körpern die Kjeldahl'sche Methode den richtigen Werth ergibt. Auch bei Histidin, dessen Constitution noch unbekannt ist, wird nach unserer Methode wohl der richtige Werth erhalten werden.

Ueber die Ursachen, wesshalb $K u t s c h e r$ und Steudel zu diesen schlechten Resultaten bezüglich der Kjeldahl'schen Methode gelangt sind, lassen sich natürlich nur Vermuthungen äussern. $\mathrm{Ob}$ zu geringe Kochdauer - wir haben mindestens eine Stunde oder noch länger erhitzt, weil bei den geringen Mengen der chemisch reinen Substanzen eine starke Bräunung der Flüssigkeit nicht eintritt und man desshalb die Entfärbung der Flüssigkeit nicht als Anbaltspunkt für das Ende der Oxydation ansehen kann - oder zu geringe Menge Schwefelsäure oder sonstige unbekannte Erscheinungen die Ursache ihrer schlechten Resultate gewesen sind, lässt sich ohne Nachprüfung, die ich aber bei einer solchen gleichmässigen Uebereinstimmung, wie Argutinsky's und meine Versuche ergeben, für unnöthig halte, nicht feststellen.

Dass die Kjeldahl'sche Methode in chemischen Laboratorien nicht in grossem Maassstabe angewandt wird, liegt nicht daran, weil 
134 Bernhard Schöndorff: Ueber die beobachtete Unsicherheit etc.

sie schlechte Resultate ergibt, sondern weil die Chemiker in vielen Fällen nicht wissen, in welcher Form der Stickstoff gebunden ist. Weil es nun feststeht, dass eine Reihe von Körpern, Nitro- und Cyanverbindungen und Nitrate erst nach besonderer Vorbereitung, Pyridin- und Chinolinderivate nur unvollständig ihren Stickstoff abgeben, so wendet der Chemiker eine Methode an, die ihm, wie die D umas'sche, auf jeden Fall den gesammten Stickstoff ergibt. 